\title{
Liste des travaux soutenus portant sur la période révolutionnaire (vers 1750 - vers 1830)
}

Annie Duprat

\section{(2) OpenEdition \\ 12 Journals}

Édition électronique

URL : https://journals.openedition.org/ahrf/1781

DOI : 10.4000/ahrf.1781

ISSN : 1952-403X

\section{Éditeur :}

Armand Colin, Société des études robespierristes

\section{Édition imprimée}

Date de publication : 1 septembre 2000

Pagination : 143-149

ISBN : 175 pages

ISSN : 0003-4436

\section{Référence électronique}

Annie Duprat, «Liste des travaux soutenus portant sur la période révolutionnaire (vers 1750 - vers 1830) ", Annales historiques de la Révolution française [En ligne], 321 | juillet-septembre 2000, mis en ligne le 21 février 2006, consulté le 24 avril 2022. URL : http://journals.openedition.org/ahrf/1781 ; DOI : https://doi.org/10.4000/ahrf.1781

Ce document a été généré automatiquement le 24 avril 2022.

Tous droits réservés 


\section{Liste des travaux soutenus portant sur la période révolutionnaire (vers 1750 - vers 1830)}

\section{Annie Duprat}

\section{CaenDEA}

1 Lecureur Bertrand, L'hôpital du Havre, fin XvIIe-début XIXe siècle, ss dir. J.-M.Moriceau, 1997.

2 Levée-Arruti Suzanne, Une recherche démographique, Tinchebray de 1700 au milieu du XIXe siècle, ss dir. P. Gouhier, 1996.

Maîtrises Bricon Stéphanie, Fermiers du sud du pays d'Auge, 1690-1830, J.-M.Moriceau, 1999.

4 Dufy Hélène, Des auteurs britanniques et la Déclaration des droits de l'homme de 1789 (1790-1796), ss dir. D. Teysseire, 1999. Haupais Murielle, La violence dans l'arrondissement de Bayeux,1796-1811, ssdir. M.Bée, 1996.

6 Léger Sylvie, Deux paroisses du Cotentin, Sainte-Mère-l'Église et Sainte-Marie-du-Mont, 1795-1830,ss dir. M. Bée, 1997.

7 Loret Donatien, La politique à Honfleur, 1791-1795, ss dir. M. Bée, 1996.

8 Poncet Fabrice, L'élevage dans la région d'Isigny (fin XVIIe-1840), ss dir. J.-M. Moriceau, 1999.

9 Quenouille Christelle, Les modes de vie de la société rurale du pays d'Auge de 1750 à 1825, ss dir J.-M. Moriceau, 1999.

10 Saumon Isabelle, Les discours de Targat aux États généraux et à l'Assemblée constituante, 1789-1791, ss dir. D. Teysseire, sd.

Dijon

11 Dollinger Sonia, Les classes dirigeantes beaunoises, 1789-1830. Changement ou continuité ? ss dir. A. Ruget, 1996.

12 Humbert Cédric, Élections et députés dans l'Yonne sous la Révolution française, ss dir. A. Ruget, 1996. 
Lancelot Mathilde, Le théâtre municipal d'Auxerre au XIXe siècle (1801-1870). Administration culturelle et lieu de sociabilité, ss dir. A. Ruget, 1996.

Morlain Marianne, Naissance d'une santé publique en Côte-d'Or (an IX-1848), ss dir. A.Ruget, 1996.

Perreau Christophe, Le personnel municipal dijonnais sous la période révolutionnaire (1789-1799), ss dir. A. Ruget, 1996.

Troussard Séverine, Situation du clergé paroissial en Côte-d'Or (1800-1848), ss dir. A. Ruget, 1996.

Bazot Marie-Laure, Le personnel municipal de la Nièvre pendant la période révolutionnaire, 1789-1799, ss dir. A. Ruget, 1997.

Baudeau Laetitia, Soldats et conscription à la fin du Premier Empire en Côte-d'Or (1810-1814), ss dir. M. Carrez, 1997.

Gouspy Cécile, Études des guides et récits de voyage sur la ville de Dijon (1792-1914), ss dir. A. Ruget, 1997.

Putin Sylvia, L'administration municipale de Cuiseaux sous la Révolution (de 1789 à juillet 1794), ss dir. A. Ruget, 1997.

Sabatier-Degrand Aude, Étude historiographique des écrits de l'avocat dijonnais Pierre Landes, de la contestation du pouvoir royal à la contre-Révolution, 1789-1790, ss dir. A. Ruget, 1997.

Decrette-Jager Nelly, Le personnel politico-administratif en Côte-d'Or sous le Directoire (1794-1799), ss dir. A. Ruget, 1998.

3 Jacob Anne-Françoise, Une famille bourguignonne face à la Révolution française les Fevret de Saint-Mémin. Exemple d'une émigration nobiliaire, ssdir. A. Ruget, 1998.

4 Juaneda Olivier, Administration impériale et conscription l'exemple de la Côte-d'Or (1810-1814),ss dir. A. Ruget, 1998.

Garcia Sonia, Administration et administrateurs du département de Saône-et-Loire sous la Révolution française (1790-1793), ss dir. A. Ruget, 1998.

Morlot Sophie, Les écoles primaires en Côte-d'Or sous la Révolution française, ss dir. Ch. Lamarre, 1999.

Tréhorel Clothilde, Le théâtre et les fêtes à Dijon sous la Révolution française, ss dir. Ch. Lamarre, 1999.

Paris I - Panthéon-SorbonneThèses

Belissa Marc, La cosmopolitique du droit des gens (1713-1795). Fraternité universelle et intérêt national au siècle des Lumières et pendant le Révolution française, ss dir. J.-P. Bertaud, 1997.

Bianchi Serge, Vie, pratiques et sociabilité politiques en milieu rural dans le sud de l'île-deFrance (Essonne et Val-de-Marne) de 1787 à 1800, doctorat d'État, ss dir. M. Vovelle, 1996.

30 Blanchard Alain, État, impôt et société. La fiscalité directe dans la généralité de Soissons au XVIIIe siècle, ss dir. J.-C. Perrot, 1997.

31 Blanckaert Jean, L'Histoire naturelle de l'homme XVIIIe-XIXe siècle, HDR, ssdir. J. SalomonBayet, 1998.

32 Bosséno Christian-Marc, Les signes extérieurs. Diffusion, réception et image de la culture révolutionnaire française dans l'Italie du Triennio, ss dir. M.Vovelle, 1996. 

en France avant et pendant la Révolution, ss dir. M.Vovelle, 1996. polymorphe, ss dir. D. Roche, 1998. XIve-fin XVIIIe siècles),ss dir. J. Jacquart, 1997. XVIIIe siècles, ss dir. É. François, 1998. phase de la révolution espagnole (1808-1810), ss dir. Guerra, 1999. siècle, ss dir. J.-C. Perrot, 1997. Révolution française ss dir. M. Vovelle, 1997. Bretagne, doctorat d'État, ss dir. D. Roche, 1998. à celle de 1830, HDR, ss dir. C. Duprat, 1996. ss dir. D. Roche, 1998. ss dir. D. Roche, 1998. de l'Empire (an VI-1815), ss dir. J.-P. Bertaud, 1998. d'interprétation, ss dir. Guerra, 1997. sociocritique (1789-1799), doctorat d'État, ss dir. M.Vovelle, 1995.

Paris X-Nanterre 1793, ss dir. A. Cabantous, 1997. Cabantous, 1997.

Brégant-Dupuy Hélène, Genèse de la patrie moderne. La naissance de l'idée moderne de patrie

Brossault-Bucas Colette, Les intendants de Franche-Comté (1674-1790), ssdir. D.Roche, 1998.

Croq Laurence, «Les Bourgeois de Paris » au XVIIIe siècle. Identification d'une catégorie sociale

Dinet Dominique, Réguliers et vie régionale dans les diocèses d'Auxerre, Langres et Dijon (fin

Duprat Annie, Les rois de papier. Études iconographiques, HDR, ss dir. D.Roche, 1998.

Gantet Claire, Discours et images de la paix dans des villes d'Allemagne du Sud aux XVIIe et

Hasquenoph Sophie, Les Dominicains de Paris au XVIIIe siècle, ss dir. D.Roche, 1997.

Hildesheimer Françoise, Richelieu : retour aux sources, HDR, ss dir. N.Lemaître, 1998.

Hocquellet Richard, Du soulèvement patriotique à la souveraineté nationale. La première

Kim Bok Rae, Les consommations alimentaires de Paris au XVIIIe et à la première moitié du XIXe

Leguillois Robert, Paris-province. Sociologie de la population parisienne pendant la

Lemaître Alain, Espace, sécurité, population au xvIIIe siècle. La police générale du parlement de

Lévêque Pierre, Les officiers de marine de Napoléon Ier, ss dir. J.-P. Bertaud, 1999.

Maréchaux Xavier, Les prêtres mariés sous la Révolution française, ss dir. M.Vovelle, 1996.

Peyrard Christine, La politique démocratique, émergence et tradition. De la génération de 1789

Picco Dominique, Les demoiselles de Saint-Cyr (1686-1793), ss dir. D. Roche, 1998.

Roy Jean-Michel, Les marchés alimentaires parisiens et l'espace urbain du XVIIe au XIXe siècle,

Sabatier Gérard, Versailles ou la figure du Roi. Essai sur le représentation du Prince en France au temps de la monarchie absolue, doctorat d'État, ssdir. D. Roche, 1996.

1 Sarrazin Véronique, Les almanachs parisiens au XVIIIe siècle. Production, commerce, culture,

Seigan Kobo, La conscription dans le département de la Seine-Inférieure du Directoire à la fin

Silva Olarte Renan, Les éclaires de Nouvelle-Grenade 1760-1808. Généalogie d'une communauté

Usandivaras-Mili Muriel, Le théâtre de la Révolution française. Étude analytique, historique et

Bouchard Stéphanie, La déchristianisation dans le district de Pontoise en novembre-décembre

Dufour Marion, La vie politique à Vendôme municipalités et notabilités, 1765-1800, ss dir. A. 

au début du Consulat, ss dir. A. Cabantous et C.Wolikow, 1998.

Poitiers d'Olonne (1791-1832), ss dir. J. Marcadé, 1995. dir. J. Péret, 1996.

Barbeau Corinne, Prénoms et parrainages à Rochefort (1688-1795), 1997. l'enregistrement de Poitiers, 1998. en Poitou dans une société pré-révolutionnaire et révolutionnaire, 1998. 1996. Cognaçais d'après les testaments (1690-1790), 1998. 1769-1790, 1997. géré par des cénobites, 1998. Convention nationale (1792-1795), ss dir. J. Marcadé, 1995. siècle, 1996. Marcadé, 1997. 1996. 1998. volumes), 1999. la seconde moitié du XVIIIe siècle, 1999.

Jarry François, L'Ami des lois. Un quotidien politique d'opinion et d'information du Directoire

Airaud Christelle, La vente des biens nationaux de première origine dans le district des Sables

Aubineau Valérie, La guerre de Vendée vue par les femmes nobles études de leurs mémoires, ss

Auger Véronique, Lussac-les-Châteaux au rythme des années: de la naissance à la descendance au XVIIIe siècle (1685-1789). Une étude démographique et généalogique, 1998.

Baudrit Catherine, Les marchands et le marché immobilier sous le Directoire dans le bureau de

Baudrit Philippe, Criminalité, justice et répression en Poitou (1785-1793) : les relations sociales

Beillon Bertrand, La chouannerie dans le district de Rochefort (1794-1799), ssdir. J. Marcadé,

Bernier Sylvain, L'évolution des mentalités religieuses et des attitudes devant la mort dans le

Bertrand Emmanuel, Étude de la religiosité des testateurs rochelais entre 1700-1741 et

Blanchon Christelle, L'abbaye de la Merci-Dieu (1650-1789) : un domaine foncier exploité et

Blandeau Olivier, L'administration départementale du Maine-et-Loire au temps de la

Boucher Richard, La vente des biens nationaux de première et seconde origine dans le bocage vendéen (districts de Montaigu et de la Châtaigneraie, 1791-1815, ss dir. J. Péret, 1996.

Charpentier Emmanuelle, Les îles de Marennes: trois justices seigneuriales à la fin du XVIIIe

Constant Magalie, Bouchemaine, un village républicain en Anjou (1789-1799), ss dir. J.

Couprie Rosemay, L'Hôpital Général et l'enfance abandonnée à Saintes, 1765-1802, 1997.

Crespeau Guillaume, Les gens de mer rochelais à travers les registres paroissiaux, 1740-1791,

74 Debiais Frédéric, Ces messieurs de Saint-Savin: l'abbaye royale de Saint-Savin, 1769-1790,

Dhenu Nathalie, Annonces, affiches et avis divers de la généralité de La Rochelle, 1772-1776, (2

Eichert Audrey, Le mariage et la vie familiale dans un espace rural : Sommières-du-Clain dans

Ferrier Fabienne, Une abbaye bénédictine à la veille de la Révolution française: l'abbaye de Saint-Cyprien de Poitiers, 1997. 

Ier. Prosopographie et stratégie de carrière dans la marine militaire de 1775 à 1815 dans l'arrondissement maritime de Rochefort. Essai de biographies comparées, 1998.

Fillion Angelina, L'abbaye de Saint-Cyprien-lès-Poitiers, déclin ou subsistance : un demi-siècle d'histoire (1746-1790), 1998.

Fricoult Magali, L'abbaye bénédictine de Saint-Junien de Nouaillé au xvIIIe siècle: une communauté de religieux de 1721 à 1790, 1998.

Gellusseau Nicolas, Un marchand droguiste à Poitiers, Jacques Farran (1775-1793), 1999.

Goubaud-Gautreau Anne, Les corsaires de Nantes pendant les guerres révolutionnaires (1793-1799) à travers l'exemple de "l'Hydre », ss dir. J.Marcadé, 1995.

Guerry Elsa, Pierre-Samuel Meschinet de Richemond: la vie d'un armateur rochelais au XVIIIe siècle (1740-1807), 1997.

Hardy Sandra, La baronnie de Chauvigny de 1672 à 1789, 1996.

Jarry Cécile, Le Journal de Poitiers et du département de la Vienne de la Terreur au sacre impérial (1793-1804), 1999.

Jonquet Corinne, La vie rurale dans le canton actuel de Champagne-Mouton de 1770 à 1789, 1996.

Lacoste Benoît, Des officiers de la marine de Louis XIV aux officiers de marine sous Napoléon Lacoste Benoît, L'activité de l'arsenal de Rochefort au travers de la correspondance du commandant Le Vassort de la Touche avec la Cour de 1775 à 1781, 1998.

88 Lascaux Sophie, La vie confolentaise à la veille de la Révolution, 1750-1789, d'après l'étude de 82 inventaires après décès, 1996.

89 Le Dû Stéphanie, Les conditions économiques et sociales des veuves de 1791 à 1797 dans le bureau de l'enregistrement de Poitiers, 1999.

Le Hénanff, L'univers des boutiquiers de Châtellerault (1752-1792) d'après l'étude de 75 inventaires, 1996.

Le Yondre Annaïg, La Terreur politique dans le district de Vannes (automne 1793-fin 1794), ss dir. J. Marcadé, 1994.

2 Leduc Sandra, L'étude testamentaire: un indicateur religieux et sociologique de la population. L'exemple du chef-boutonnais à la veille de la Révolution, 1650-1793, 1999.

3 Lemaire Delphine, La vie de la noblesse poitevine au Xville siècle d'après les inventaires après décès, 1996.

94 Lemerige Béatrice, Les attitudes devant la mort à Châtellerault de 1780 à 1805, à travers les testaments, 1995.

5 Leroy Laurent, Trois tests du comportement religieux dans onze paroisses du pays mélusin (1780-1792), 1997.

Louet Mélika, Vivre au pays montois au XVIIIe siècle (canton actuel de Monts-sur-Guesne), 1998.

9 Magneron Hélène, Catholiques et protestants à Lezay. Étude démographique et religieuse (1677-1789), 1998.

98 Marais Arnaud, Les « réfugiés de la Vendée » en Charente, 1997.

Martin Sandra, Les itinéraires mouvementés de deux prêtres poitevins de la fin du xVIIIe siècle au début du XIxe siècle: François Guillemot (1750-1833) et Alexandre Guillemot (1757-1843), 1999. 


$$
1999 .
$$

Rennes II 


$$
1998 .
$$

ToursDEA

\section{Maîtrises}

Desmot Nathalie, La vie quotidienne des hospices civils de Fougères (1789-1830), ss dir. J. Quéniart, 1995.

Faijean Sophie, Les suspectes en Ille-et-Vilaine entre passivité et activisme. La contreRévolution au féminin, ss dir. D. Godineau, 1996.

Gautier Stéphane, Les comités révolutionnaires dans le district de Montfort, ss dir. D. Godineau, 1998.

Le Goff Loïc, Vive Dieu et le Roy La chouannerie dans les cinq districts des Côtes-du-Nord, ss dir. R. Dupuy, 1996.

Le Liard Isabelle, La vente des livres de rebut effectuée à la Bibliothèque municipale de Rennes de 1794 à 1848, ss dir. N. Richard, 1997.

Le Roy Céline, La chouannerie en Finistère (1793-1800), ss dir. R. Dupuy, 1995.

Meaude Martial, Sol de Grisolles, de Siz, Jean Brohan la chouannerie dans le sud-est du Morbihan (1793-1800), ss dir. R. Dupuy, 1995.

Millot Nicolas, La guerre de course au port de Lorient sous la Révolution et l'Empire (1793-1814) d'après les archives du Tribunal de commerce, ssdir. G. Le Bouédec, 1995.

Peneau Yvan, Lorient en l'an II de la République, ss dir. R. Dupuy, 1995.

Senand Ludovic, La société Joseph Gaudin fils et compagnie à Nantes (1785-1814) la manufacture de Belair, la forge de la Verrière et le fourneau du Pas-Chevreuil, ss dir. A. Geslin,

Landais Frédérique, Appartenance locale, appartenance nationale, appartenance européenne la noblesse du Loir- et-Cher de 1800 à 1848, ss dir. Cl.-Is. Brelot, 1994.

Skrobala Denis, René Gastellier (1741-1821) médecin et maire de Montargis: une pratique médicale et hygiéniste en Gâtinais à la fin de l'Ancien Régime, ssdir. C. Petitfrère, 1997.

Alphonse Christèle, L'administration des haras et l'élevage du cheval dans la généralité de Tours, 1749-1789, ss dir. N. Coquery, 1998.

Bienassis Loïc, L'idée et les représentations de la noblesse dans l'œuvre de Châteaubriand, ss dir. Cl-Is. Brelot, 1995

Blas Delphine, Étude démographique des paroisses de Nouan-sur-Loire et de Saint-Laurent-desEaux de 1741 à 1792, ss dir. B. Maillard, 1997.

Boisard Corinne, La vie quotidienne des paysans en Touraine du Nord de 1766 à 1806, ss dir. B. Maillard, 1995.

Bucas Xavier, La paroisse de Notre-Dame de Bonneval dans la deuxième moitié du XVIIIe siècle, ss dir. B. Maillard, 1996.

Bureau Marie-Anne, La paroisse de Ballan-Miré au xvIIIe siècle. Étude démographique, 1736-1795, ss dir. B. Maillard, 1996.

Bury Jean-Yves, La Garde nationale de Blois au début de la Révolution (1789-janvier 1792), ss dir. C. Petitfrère, 1995.

Chin Man-Yi, Les lettres de Manon Phlipon à Sophie Canet contribution à l'histoire de l'amitié au XvIIIe siècle, ss dir. C. Petitfrère, 1997.

4 Coisnon Thibaut, La municipalité de Tours au début de la République, ss dir. C. Petitfrère, 1997. 
Courson Danaé, Le pouvoir politique au village Charentilly (1800-1852), ssdir. Cl-Is. Brelot, 1994.

Cousin Isabelle, Monographie paroissiale Notre-Dame de Paradis-Hennebont (1726-1745 / 1771-1790). Étude démographique et intérieurs hennebontais, ss dir. B. Maillard, 1995.

Djambourian Karen, La justice civile au bailliage et siège présidial de Tours (1770-1773), ss dir. C. Petitfrère, 1995.

Fourneau Hélène, La paroisse de Neuillé-Pont-Pierre au XvIIIe siècle (1737-1792), ss dir. B. Maillard, 1995.

Gouffier Hervé, Naître, vivre et mourir à Savigny (1701-1792). Étude d'un isolat démographique au XVIIIe siècle, ss dir. B. Maillard, 1997.

Gracet Xavier, La noblesse et ses relations avec les autres couches de la société à travers les archives de justice de paix d'Amboise (1814-1852), ss dir.Cl-Is.Brelot, 1996.

Guenand Agathe, La fabrique des poudres et salpêtres du Ripault (1786-1817), ss dir. B. Maillard, 1998.

Guérin-Boutaud Cécile, Le corps de ville d'Angoulême dans la deuxième moitié du xvIIIe siècle (1751-1789), ss dir. C. Petitfrère, 1997.

Hiault Emmanuel, Les cahiers de doléances des corps et communautés de métiers à Tours en 1789, ss dir. N. Coquery, 1998.

Jarraud Isabelle, Les compagnons du Devoir à Tours à la fin du XviIIe siècle 1792-1802, ss dir. B. Maillard, 1995.

Jeannin Emmanuelle, Une perception anglaise de la France Mary Wollstonecraft et l'image de la France en période révolutionnaire, ss dir. G.Chaix et P. Brioist, 1996.

Martin Laurent, La violence dans le village de Chinon d'après les archives judiciaires (1779-1789), ss dir. B. Maillard, 1997.

Pelletier Delphine, Le couvent des Ursulines de Tours sous l'Ancien Régime, 1622-1792, ss dir. G. Chaix, 1998.

Pierron-Lévêque Hélène, Le tribunal criminel du Cher de 1792 à Thermidor an II. L'institution et les hommes, ss dir. C. Petitfrère, 1995.

Pinchault Laurent, Imprimeurs-libraires, imprimés et bibliothèques à Blois ausiècle des Lumières (1720-1790), ss dir. G. Chaix, 1996.

60 Poupin Delphine, Nobles et ruraux en Touraine à travers les archives de justice de paix des cantons d'Azay-le-Rideau et de Sainte-Maure de Touraine, entre 1822 et 1848, ss dir. Cl-Is. Brelot, 1995.

Rénier Olivier, Les compagnons des corporations de Tours dans les années 1780, ss dir. N. Coquery, 1998.

62 Saavedra-Pugnière Isabel, Toussaint-Bernard Émeric-David (1755-1819), leVasari français, ss dir. D. Poulot, 1999.

Salic Delphine, Le maintien de l'ordre à Tours d'après les archives du lieutenant de police (1775-1784), ss dir. B. Maillard, 1995. 